Article

\title{
Asymptotic Tail Probability of the Discounted Aggregate Claims under Homogeneous, Non-Homogeneous and Mixed Poisson Risk Model
}

\author{
Franck Adékambi *D and Kokou Essiomle
}

Schoool of Economics, University of Johannesburg, Johannesburg 2006, South Africa; 201900490@student.uj.ac.za

* Correspondence: fadekambi@uj.ac.za

\begin{abstract}
In this paper, we derive a closed-form expression of the tail probability of the aggregate discounted claims under homogeneous, non-homogeneous and mixed Poisson risk models with constant force of interest by using a general dependence structure between the inter-occurrence time and the claim sizes. This dependence structure is relevant since it is well known that under catastrophic or extreme events the inter-occurrence time and the claim severities are dependent.
\end{abstract}

Keywords: homogeneous; non-homogeneous; mixed Poisson risk model; differential equation; discounted aggregate loss; subexponential; tail probability

MSC: 2010; 62P05; 97K60

Citation: Adékambi, Franck, and Kokou Essiomle. 2021. Asymptotic Tail Probability of the Discounted Aggregate Claims under Homogeneous, Non-Homogeneous and Mixed Poisson Risk Model. Risks 9: 122 https://doi.org/10.3390/risks 9070122

Academic Editor: Mogens Steffensen

Received: 28 April 2021

Accepted: 2 June 2021

Published: 30 June 2021

Publisher's Note: MDPI stays neutra with regard to jurisdictional claims in published maps and institutional affiliations.

Copyright: (c) 2021 by the authors. Licensee MDPI, Basel, Switzerland. This article is an open access article distributed under the terms and conditions of the Creative Commons Attribution (CC BY) license (https:// creativecommons.org/licenses/by/ $4.0 /$ )

\section{Introduction}

In actuarial science, the discounted sum of losses over time can be considered as a randomly weighted sum of a sequence of independent and identically distributed (i.i.d.) random variables. Those independent and identically distributed random variables represent the claim amounts in successive development years, while the weights represent the discounted factors. Similar studies that have assumed independence between the inter-occurrence time and the forthcoming claim amounts include Taylor (1979), Delbaen and Haezendonck (1987), Waters (1983), Sundt and Teugels (1995), Cai (2002) and Yuen et al. (2006) who not only assumed independence between inter-occurrence time but have also derived the asymptotic tail probabilities associated with free interest risk model. In addition, Boogaert et al. (1988), Willmot (1989), Leveillé and Garrido (2001a, 2001b), Leveillé and Adékambi $(2011,2012)$ and Léveillé et al. (2010) have not only assumed the independence between the inter-occurrence time and the claim sizes but have also generalized the moments of the aggregate renewal sums. Leveille and Hamel (2018) have used non-homogeneous and doubly compound Poisson process with stochastic force of interest and have derived the moments generating functions of the discounted aggregate claims. Moreover, they have found the inverse of the moments generating functions numerically and analytically. Geluk and Tang (2009) have studied the asymptotic behavior of the tail probabilities of a sum of real-value random variables. They have assumed that the distributions of the random variables belong to the subexponential class. Using asymptotically independence assumptions, they have showed that the asymptotic behavior of the tail probabilities is the same as that of the independence case. Although the independence assumption appears to be custom in risk theory, it has recently been subject to severe criticism due to the fact that it does not provide reliable estimates under extreme events, for example, see Boudreault et al. (2006) and Zhang and Yang (2011). Thus, the use of dependent risk models has exhibited some positive interests in the literature. Yang et al. (2012) have also studied the asymptotic behavior of the tail probabilities of a randomly weighted sum of subexponential random variables under dependence structure. In their 
paper, they have assumed that the random weights and the corresponding summand are dependent and the sequence of the pairs of random variables are i.i.d. Liu and Gao (2015) have considered the problem of the tail behavior of the discounted aggregate sum in dependent risk models with constant force of interest. They have assumed that the claim sizes are of an upper tail asymptotic independence structure. Moreover, they have assumed that the claim sizes and the inter-occurrence time have a specific dependence structure and they have derived an asymptotic formula in the case where the claim size distribution belongs to the intersection of long-tailed distribution class and dominant variation class. Albrecher and Boxma $(2004,2005)$ have developed a dependent risk model in which they have assumed that the inter-claim time and the forthcoming claim amounts are governed by a semi-Markov process. Further to that, Albrecher and Teugels (2006) have used a random walk process to model the dependency between the inter-claim time and the forthcoming claim amounts. Their results are more elaborate as they have modeled this dependency using a copula and have derived exponential estimates of the ruin probabilities in finite and infinite time horizons. Yang et al. (2014) have considered a nonstandard risk model in which they have assumed a Lévy process for the interest rate. Assuming a specific dependency among the claims, they have derived the tail probability of the discounted aggregate claim. Lu et al. (2018) have extended the work of Yang et al. (2014) by considering the multi-risk model. They incorporated the dependence structure among the claim severities and by assuming a multidimensional Lévy process for the force of interest, they have derived the asymptotic tail distribution of the discounted aggregate claim. This paper attempts to assess the impact of the dependency between the inter-occurrence time and the forthcoming claim severities through a copula approach and derives the asymptotic tail probabilities. The closest study to ours is that of Asimit and Badescu (2010) who have considered in a constant force of interest environment a heavy tailed distribution of the discounted aggregate claim in which the dependence structure is modeled via copulas. They have derived numerically the asymptotic tail probabilities, the asymptotic finite time ruin probabilities and asymptotic approximations for some common risk measures associated with the discounted aggregate claims distribution, without taking into account any effect of the interest rate. Asimit and Badescu (2010) have also derived a convolution form of the asymptotic tail probability of the aggregate claim under homogeneous Poisson risk model when the force of interest rate $\delta \neq 0$ and a closed-form expression has only been derived for the special case of $\delta=0$. In the present paper, we have extended their result by finding a closed-form expression of the tail probability under homogeneous, mixed and non-homogeneous Poisson processes (Theorems 2-7), assuming that the counting process following Poisson distribution may be restrictive.

The model inputs are set as follows:

- $\quad$ The counting processes $\{\mathrm{N}(t), t \geq 0\},\left\{\mathrm{N}_{n h}(t), t \geq 0\right\}$ and $\left\{\mathrm{N}_{m h}(t), t \geq 0\right\}$ form respectively homogeneous, non-homogeneous and mixed Poisson processes.

- Non-negative random variables $\left\{V_{k}, k \in \mathbb{N}\right\}$ that represent the inter-occurrence times.

- Non-negative random variables $\left\{T_{k}, k \in \mathbb{N}\right\}$ such that $T_{k}=\sum_{j=1}^{k} V_{j}$, represent the claim occurrence times.

- $\quad$ Positive i.i.d random variables $\left\{B_{k}, k \in \mathbb{N}\right\}$ that represent the claim severities. It is assumed that $\left\{B_{k}, V_{k}, k \in \mathbb{N}-1\right\}$ are independent of $\left\{B_{1}, V_{1}\right\}$.

The discounted aggregate claim process for homogeneous, non-homogeneous or mixed Poisson models over a finite time horizon $(0 ; t]$ is given by

$$
Z_{\delta}(t)=\sum_{k=1}^{N(t)} B_{k} e^{-\delta T_{k}}, \quad Z_{\delta}^{n h}(t)=\sum_{k=1}^{N_{n h}(t)} B_{k} e^{-\delta T_{k}}, Z_{\delta}^{m h}(t)=\sum_{k=1}^{N_{m h}(t)} B_{k} e^{-\delta T_{k}},
$$

where $Z_{\delta}(t)=Z_{\delta}^{n h}(t)=Z_{\delta}^{m h}(t)=0$ if $N(t)=N_{n h}(t)=N_{m h}(t)=0$ and $\delta$ is the force of interest.

The remaining is structured as follows. Section 2 reviews the type of dependence structure used in the study. Section 3 gives a closed-form expression of the tail probability 
for the homogeneous, non-homogeneous and mixed Poisson risk models and finally in Section 4 we apply these results to derive some risk measures such as the asymptotic finite time ruin probability and the value-at-risk and also provide some numerical illustrations for these risk measures.

\section{Preliminaries}

This paper considers the same dependence structure for the $k$-th inter-occurrence time $V_{k}$ and its corresponding claim size $B_{k}$ as in Asimit and Badescu (2010) and derived the tail probability for the aggregate claim.

The following assumption (from Asimit and Badescu (2010)) characterizes the type of dependence structure under study.

Assumption 1. The pairs of random variables $\left(V_{i}, B_{i}\right), i=1,2, \ldots$ are mutually i.i.d. Moreover, there exists a positive and locally bounded function $l()$ such that the relation

$$
\operatorname{Pr}\left(B_{1}>b \mid V_{1}=v_{1}\right) \sim \operatorname{Pr}\left(B_{1}>b\right) l\left(v_{1}\right),
$$

holds uniformly for all $v_{1} \in(0, T]$ as $b \rightarrow+\infty$ where $T$ is the time horizon.

Here uniformly is understood as

$$
\lim _{b \rightarrow \infty} \sup _{v \in(0, t]}\left|\frac{\operatorname{Pr}\left(B_{1}>b \mid V_{1}=v_{1}\right)}{\operatorname{Pr}\left(B_{1}>b\right) l\left(v_{1}\right)}-1\right|=0 .
$$

As in Asimit and Badescu (2010), Assumption 1 is motivated by the fact that under its premises, one can study a wide class of dependence structure given in terms of a copula.

From Sklar's theorem (see Sklar 1959), if $H$ is a joint cumulative distribution function with continuous marginals $H_{1}$ and $H_{2}$ respectively, there exists a unique copula $C$ such that

$$
H(b, v)=C\left(H_{1}(b), H_{2}(v)\right),(b, v) \in \operatorname{Dom}(H) .
$$

So, for example, the survival copula is defined as the copula relative to the joint survival function and is given by

$$
\hat{C}\left(u_{1}, u_{2}\right)=u+v-1+C\left(1-u_{1}, 1-u_{2}\right),\left(u_{1}, u_{2}\right) \in[0,1]^{2} .
$$

For more details on the theory of copulas, the reader is referred to the book of Nelsen (1999).

It follows (see Asimit and Badescu 2010) that if $\hat{C}_{2}\left(u_{1}, u_{2}\right)=\frac{\partial \hat{C}\left(u_{1}, u_{2}\right)}{\partial u_{2}}$ exists, then Assumption 1 can be rewritten as

$$
\lim _{u_{1} \downarrow 0} \sup _{u_{2} \in\left[e^{-\lambda T}, 1\right)}\left|\frac{\hat{C}_{2}\left(u_{1}, u_{2}\right)}{u_{1} h\left(u_{2}\right)}-1\right|=0, \text { where } h(v):=l\left(\frac{-\log v}{\lambda}\right) .
$$

In this paper, we consider only the case of heavy-tailed claim size distributions. Let us denote by $F$ the cumulative distribution function of a non-negative random variable $B, \bar{F}$ its tailed distribution and $\overline{F^{* n}}$ the n-fold convolution of $\bar{F}$

(1) The distribution of $B$ is said to belong to the subexponential class $(\mathcal{S})$ if and only if

$$
\lim _{b \rightarrow \infty} \frac{\overline{F^{* 2}}(b)}{\bar{F}(b)}=2 .
$$


(2) The distribution of $B$ is said to belong to regularly varying class $\left(R V_{-\alpha}\right)$ if and only if

$$
\lim _{b \rightarrow \infty} \frac{\bar{F}(b y)}{\bar{F}(b)}=y^{-\alpha}, \alpha>0
$$

For more information on heavy-tailed distributions and its applications, the reader is referred to Su et al. (2007) and references therein.

\section{Asymptotic Expression of the Cumulative Distribution of $\mathrm{Z}_{\delta}(T)$}

Recall from Section 1 that $Z_{\delta}(T)$ is the random variable that represents the discounted aggregate claim process. If the actuary knows the form of $Z_{\delta}(T)$, then he can apply the quantile principle to calculate the premium or risk measures such as the ruin probability or the value-at-risk.

\subsection{Homogeneous Poisson Risk Process}

Let us recall the following theorem from Asimit and Badescu (2010) for the tail probability of $\mathrm{Z}_{\delta}(t)$.

Theorem 1. Consider a homogeneous compound Poison risk process with constant force of interest $\delta>0$ such that the cumulative function $(F)$ of $B$ belongs to $R V_{-\alpha}$. If Assumption 1 is satisfied for any $t \in(0 ; T], T$ is time horizon, then

$$
\mathbf{P}\left(\mathrm{Z}_{\delta}(T)>b\right) \sim K_{\delta}(T) \mathbf{P}\left(\mathrm{B}_{1}>b\right), \quad b \longrightarrow+\infty,
$$

where

$$
K_{\delta}(T)=e^{-\lambda T} \sum_{n=1}^{\infty} \lambda^{n} \int_{0}^{T} \int_{0}^{v_{n}} \cdots \int_{0}^{v_{2}} \sum_{i=1}^{n} l\left(v_{i}\right) e^{-\alpha \delta \sum_{j=1}^{i} v_{j}} d v_{1} \cdots d v_{n} .
$$

Theorem 1 gives the convolution form of the tail probability of the aggregate claim. Although this result is interesting, it has a drawback as a closed analytical expression is still unknown, thus the multiple integrals need to be evaluated numerically and may cost in time. To address the issue, the following theorems derive and solve the differential equation of $K_{\delta}(T)$ given above.

Theorem 2. Under the assumption of Theorem 1, the deterministic function $K_{\delta}(T)$ satisfied the following differential equation

$$
\frac{\mathrm{d}^{2} K_{\delta}(T)}{\mathrm{d} T^{2}}=\left(\lambda e^{-\alpha \delta T}-\alpha \delta-\lambda\right) \frac{\mathrm{d} K_{\delta}(T)}{\mathrm{d} T}+\lambda l^{\prime}(T) e^{-(\alpha \delta+\lambda) T}
$$

where $K_{\delta}(0)=0$ and $\left.\frac{\partial K_{\delta}(T)}{\partial T}\right|_{T=0}=\lambda l(0)$.

Remark 1. If $\delta=0$ then

$$
\frac{\mathrm{d}^{2} K_{0}(T)}{\mathrm{d} T^{2}}=\lambda l^{\prime}(T) e^{-\lambda T}
$$

The solution of this differential equation is consistent with the result of Asimit and Badescu (2010). 
Proof. From Equation (3) of Theorem 1 we have for any $T \geq 0$,

$$
\begin{aligned}
K_{\delta}(T) & =e^{-\lambda T} \sum_{n=1}^{\infty} \lambda^{n} \int_{D_{n}} \sum_{i=1}^{n} l\left(v_{i}\right) e^{-\alpha \delta \sum_{j=1}^{i} v_{j}} d v \\
& =e^{-\lambda T} \sum_{n=1}^{\infty} \lambda^{n} \int_{0}^{T} \int_{0}^{v_{n}} \cdots \int_{0}^{v_{2}} \sum_{i=1}^{n} l\left(v_{i}\right) e^{-\alpha \delta \sum_{j=1}^{i} v_{j}} d v_{1} \cdots d v_{n} \\
& =e^{-\lambda T} \sum_{n=1}^{\infty} \lambda^{n} \int_{0}^{T} \int_{0}^{v_{n}} \cdots \int_{0}^{v_{2}} \sum_{i=1}^{n} l\left(v_{i}\right) \prod_{j=1}^{i} e^{-\alpha \delta v_{j}} d v_{1} \cdots d v_{n} \\
& =e^{-\lambda T}\left\{\int_{0}^{T} \lambda l(v) e^{-\alpha \delta v} d v+\sum_{n=2}^{\infty} \lambda^{n} \int_{0}^{T} \int_{0}^{v_{n}} \cdots \int_{0}^{v_{2}} \sum_{i=1}^{n} l\left(v_{i}\right) e^{-\alpha \delta \sum_{j=1}^{i} v_{j}} d v_{1} \cdots d v_{n}\right\},
\end{aligned}
$$

where, at $n=1$, the preceding notations mean that we integrate only between 0 and $T$.

By taking the first derivative of the preceding expression with respect to $T$ we have,

$$
\begin{aligned}
\frac{\mathrm{d} K_{\delta}(T)}{\mathrm{d} T} & =-\lambda K_{\delta}(T)+e^{-\lambda T} \lambda l(T) e^{-\alpha \delta T}+e^{-\lambda T} \lambda \sum_{n=2}^{\infty} \lambda^{n-1} \int_{0}^{T} \int_{0}^{v_{n-1}} \\
& \cdots \int_{0}^{v_{2}}\left[\sum_{i=1}^{n-1} l(v) \prod_{j=1}^{i} e^{-\alpha \delta v_{j}}+l(v) e^{-\alpha \delta \sum_{j=1}^{n-1} v_{j}} e^{-\alpha \delta T}\right] d v_{1} \cdots d v_{n-1} \\
& =-\lambda K_{\delta}(T)+\lambda l(T) e^{-(\alpha \delta+\lambda) T} \\
& +e^{-\lambda T} \lambda \sum_{n=2}^{\infty} \lambda^{n-1} \int_{0}^{T} \int_{0}^{v_{n-1}} \cdots \int_{0}^{v_{2}} \sum_{i=1}^{n-1} l(v) \prod_{j=1}^{i} e^{-\alpha \delta w_{j}} d v_{1} \cdots d v_{n-1} \\
& +e^{-(\alpha \delta+\lambda) T} \lambda \sum_{n=2}^{\infty} \lambda^{n-1} \int_{0}^{T} \int_{0}^{v_{n-1}} \cdots \int_{0}^{v_{2}} l(v) e^{-\alpha \delta \sum_{j=1}^{n-1} v_{j}} d v_{1} \cdots d v_{n-1}
\end{aligned}
$$

Rearranging this expression gives,

$$
\begin{aligned}
\frac{\mathrm{d} K_{\delta}(T)}{\mathrm{d} T} & =-\lambda K_{\delta}(T)+\lambda l(T) e^{-(\alpha \delta+\lambda) T} \\
& +e^{-\lambda T} \lambda \sum_{n=1}^{\infty} \lambda^{n} \int_{0}^{T} \int_{0}^{v_{n}} \cdots \int_{0}^{v_{2}} \sum_{i=1}^{n} l(v) \prod_{j=1}^{i} e^{-\alpha \delta v_{j}} d v_{1} \cdots d v_{n} \\
& +e^{-(\alpha \delta+\lambda) T} \lambda \sum_{n=1}^{\infty} \lambda^{n} \int_{0}^{T} \int_{0}^{v_{n}} \cdots \int_{0}^{v_{2}} l(v) e^{-\alpha \delta \sum_{j=1}^{n} v_{j}} d v_{1} \cdots d v_{n} \\
& =-\lambda K_{\delta}(T)+\lambda l(T) e^{-(\alpha \delta+\lambda) T}+\lambda K_{\delta}(T) \\
& +e^{-(\alpha \delta+\lambda) T} \lambda \sum_{n=1}^{\infty} \lambda^{n} \int_{0}^{T} \int_{0}^{v_{n}} \cdots \int_{0}^{v_{2}} l(v) e^{-\alpha \delta \sum_{j=1}^{n} v_{j}} d v_{1} \cdots d v_{n} \\
& =\lambda e^{-(\alpha \delta+\lambda) T}\left\{l(T)+\sum_{n=1}^{\infty} \lambda^{n} \int_{0}^{T} \int_{0}^{v_{n}} \cdots \int_{0}^{v_{2}} l(v) e^{-\alpha \delta \sum_{j=1}^{n} v_{j}} d v_{1} \cdots d v_{n}\right\} .
\end{aligned}
$$

As previously, the derivative of the preceding expression with respect to $T$ yields,

$$
\begin{aligned}
\frac{\mathrm{d}^{2} K_{\delta}(T)}{\mathrm{d} T^{2}} & =-(\alpha \delta+\lambda) \frac{\mathrm{d} K_{\delta}(T)}{\mathrm{d} T}+\lambda e^{-(\alpha \delta+\lambda) T}\left\{l^{\prime}(T)+\lambda l(T) e^{-\alpha \delta T}\right. \\
& \left.+\lambda \sum_{n=2}^{\infty} \lambda^{n-1} \int_{0}^{T} \int_{0}^{v_{n-1}} \cdots \int_{0}^{v_{2}} l(v) e^{-\alpha \delta \sum_{j=1}^{n-1} v_{j}} e^{-\alpha \delta T} d v_{1} \cdots d v_{n-1}\right\} \\
& =-(\alpha \delta+\lambda) \frac{\mathrm{d} K_{\delta}(T)}{\mathrm{d} T}+\lambda e^{-(\alpha \delta+\lambda) T}\left\{l^{\prime}(T)+\lambda l(T) e^{-\alpha \delta T}\right. \\
& \left.+\lambda e^{-\alpha \delta T} \sum_{n=1}^{\infty} \lambda^{n} \int_{0}^{T} \int_{0}^{v_{n}} \cdots \int_{0}^{v_{2}} l(v) e^{-\alpha \delta \sum_{j=1}^{n} v_{j}} d v_{1} \cdots d v_{n}\right\}
\end{aligned}
$$


From Equation (5) we have,

$$
\lambda e^{-\alpha \delta T} \sum_{n=1}^{\infty} \lambda^{n} \int_{0}^{T} \int_{0}^{v_{n}} \cdots \int_{0}^{v_{2}} l(v) e^{-\alpha \delta \sum_{j=1}^{n} v_{j}} d v_{1} \cdots d v_{n}=e^{\lambda T} \frac{\mathrm{d} K_{\delta}(T)}{\mathrm{d} T}-\lambda l(T) e^{-\alpha \delta T} .
$$

Then, substituting (7) into (6) leads to,

$$
\begin{aligned}
\frac{\mathrm{d}^{2} K_{\delta}(T)}{\mathrm{d} T^{2}} & =-(\alpha \delta+\lambda) \frac{\mathrm{d} K_{\delta}(T)}{\mathrm{d} T}+\lambda e^{-(\alpha \delta+\lambda) T}\left\{l^{\prime}(T)+\lambda l(T) e^{-\alpha \delta T}+e^{\lambda T} \frac{\mathrm{d} K_{\delta}(T)}{\mathrm{d} T}-\lambda l(T) e^{-\alpha \delta T}\right\} \\
& =-(\alpha \delta+\lambda) \frac{\mathrm{d} K_{\delta}(T)}{\mathrm{d} T}+\lambda e^{-(\alpha \delta+\lambda) T}\left\{l^{\prime}(T)+e^{\lambda T} \frac{\mathrm{d} K_{\delta}(T)}{\mathrm{d} T}\right\} \\
& =-(\alpha \delta+\lambda) \frac{\partial K_{\delta}(T)}{\partial T}+\lambda l^{\prime}(T) e^{-(\alpha \delta+\lambda) T}+\lambda e^{-\alpha \delta T} \frac{\mathrm{d} K_{\delta}(T)}{\mathrm{d} T} \\
& =\left(\lambda e^{-\alpha \delta T}-\alpha \delta-\lambda\right) \frac{\mathrm{d} K_{\delta}(T)}{\mathrm{d} T}+\lambda l^{\prime}(T) e^{-(\alpha \delta+\lambda) T},
\end{aligned}
$$

which proves the statement.

The next theorem gives an analytical expression of $K_{\delta}(T)$ in a homogeneous Poisson risk model.

Theorem 3. Under the assumption of Theorem 2, if we assume further that $K_{\delta}^{\prime}(t)>0$ for every $t \in[0, T]$, then the solution of the differential equation given by expression (4) of Theorem 2 is

$$
\boldsymbol{K}_{\delta}(T)=\int_{0}^{T} e^{-\left(\frac{\lambda}{\alpha \delta} e^{-\alpha \delta v}+(\alpha \delta+\lambda) v\right)} \mathrm{d} v+\lambda \int_{0}^{T} l(v) e^{-(\alpha \delta+\lambda) v}(1+(\alpha \delta+\lambda)(T-v)) \mathrm{d} v,
$$

where

$$
\lim _{\delta \rightarrow 0} \frac{\lambda}{\alpha \delta} e^{-\alpha \delta v}=+\infty
$$

Proof.

(1) Firstly let us solve Equation (4) of Theorem 2 without the second member.

$$
\text { From } \frac{\mathrm{d}^{2} K_{\delta}(T)}{\mathrm{d} T^{2}}=\left(\lambda e^{-\alpha \delta T}-\alpha \delta-\lambda\right) \frac{\mathrm{d} K_{\delta}(T)}{\mathrm{d} T}
$$

we have,

$$
\begin{aligned}
\frac{\mathrm{d}}{\mathrm{d} T}\left(\ln \left[\frac{\mathrm{d} L_{\delta}(T)}{\mathrm{d} T}\right]\right) & =\lambda e^{-\alpha \delta T}-\alpha \delta-\lambda \\
\ln \left[\frac{\mathrm{d} L_{\delta}(T)}{\mathrm{d} T}\right] & =-\left(\frac{\lambda}{\alpha \delta} e^{-\alpha \delta T}+(\alpha \delta+\lambda) T\right)+\mu_{0} \\
\frac{\mathrm{d} L_{\delta}(T)}{\mathrm{d} T} & =e^{-\left(\frac{\lambda}{\alpha \delta} e^{-\alpha \delta T}+(\alpha \delta+\lambda) T\right)}+\mu_{1} \\
L_{\delta}(T) & =\int_{0}^{T} e^{-\left(\frac{\lambda}{\alpha \delta} e^{-\alpha \delta v}+(\alpha \delta+\lambda) v\right)} \mathrm{d} v+\mu .
\end{aligned}
$$

By letting $\mu=0$ we have,

$$
L_{\delta}(T)=\int_{0}^{T} e^{-\left(\frac{\lambda}{\alpha \delta} e^{-\alpha \delta v}+(\alpha \delta+\lambda) v\right)} \mathrm{d} v .
$$

(2) Secondly let us find the solution for the second member of Equation (4). 
Let for any $T \geq 0$,

$$
H(T)=\lambda \int_{0}^{T} l(v) e^{-(\alpha \delta+\lambda) v}(1+(\alpha \delta+\lambda)(T-v)) \mathrm{d} v .
$$

One can easily check that

$$
\frac{\mathrm{d}^{2} H(T)}{\mathrm{d} T^{2}}=\lambda l^{\prime}(T) e^{-(\alpha \delta+\lambda) T} .
$$

Therefore, the final solution is

$$
\mathbf{K}_{\delta}(T)=L_{\delta}(T)+H(T),
$$

which proves the statement.

Remark 2. In the case of a mixed Poisson compound model, the differential equation of $K_{\delta}(T)$ given $\Lambda=\lambda$ is again given by Equation (4)

We can now state the theorem that gives the closed-form expression of $K_{\delta}(T)$ in a mixed Poisson compound risk model.

Theorem 4. Consider a mixed Poisson compound risk model. Assume that the intensity rate $\Lambda$ is a continuous positive random variable such that its moment generating function $\mathbf{M}_{\Lambda}(s)$ exists then, the closed-form expression of $K_{\delta}(T)$ is given by

$$
K_{\delta}(T)=\int_{0}^{T}\left\{\mathbf{M}_{\Lambda}\left(-v-\frac{e^{-\alpha \delta v}}{\alpha \delta}\right) e^{-\alpha \delta v}+\mathbf{E}\left[l(v) e^{-(\alpha \delta+\Lambda) v} \Lambda(1+(\alpha \delta+\Lambda)(T-v))\right]\right\} \mathrm{d} v .
$$

Proof. From Theorem 3, we have

$$
\left.\mathbf{K}_{\delta}(T)\right|_{\Lambda=\lambda}=\int_{0}^{T}\left\{e^{-\left(\frac{\lambda}{\alpha \delta} e^{-\alpha \delta v}+(\alpha \delta+\lambda) v\right)}+\lambda l(v) e^{-(\alpha \delta+\lambda) v}(1+(\alpha \delta+\lambda)(T-v))\right\} \mathrm{d} v,
$$

therefore,

$$
\mathbf{K}_{\delta}(T)=\mathbf{E}\left[\int_{0}^{T}\left\{e^{-\left(\frac{\Lambda}{\alpha \delta} e^{-\alpha \delta v}+(\alpha \delta+\Lambda) v\right)}+\Lambda l(v) e^{-(\alpha \delta+\Lambda) v}(1+(\alpha \delta+\Lambda)(T-v))\right\} \mathrm{d} v\right],
$$

and using Fubini's theorem, we can interchange the expectation and the integral.

Hence,

$$
\begin{aligned}
\mathbf{K}_{\delta}(T) & =\int_{0}^{T}\left\{\mathbf{E}\left[e^{-\left(\frac{\Lambda}{\alpha \delta} e^{-\alpha \delta v}+(\alpha \delta+\Lambda) v\right)}\right]+\mathbf{E}\left[\Lambda l(v) e^{-(\alpha \delta+\Lambda) v}(1+(\alpha \delta+\Lambda)(T-v))\right]\right\} \mathrm{d} v \\
& =\int_{0}^{T}\left\{\mathbf{E}\left[e^{-\left(\frac{e^{-\alpha \delta v}}{\alpha \delta} \Lambda+\Lambda v\right)}\right] e^{-\alpha \delta v}+\mathbf{E}\left[\Lambda l(v) e^{-(\alpha \delta+\Lambda) v}(1+(\alpha \delta+\Lambda)(T-v))\right]\right\} \mathrm{d} v \\
& =\int_{0}^{T}\left\{\mathbf{E}\left[e^{-\left(\frac{e^{-\alpha \delta v}}{\alpha \delta}+v\right) \Lambda}\right] e^{-\alpha \delta v}+\mathbf{E}\left[l(v) e^{-(\alpha \delta+\Lambda) v} \Lambda(1+(\alpha \delta+\Lambda)(T-v))\right]\right\} \mathrm{d} v \\
& =\int_{0}^{T}\left\{\mathbf{M}_{\Lambda}\left(-v-\frac{e^{-\alpha \delta v}}{\alpha \delta}\right) e^{-\alpha \delta v}+\mathbf{E}\left[l(v) e^{-(\alpha \delta+\Lambda) v} \Lambda(1+(\alpha \delta+\Lambda)(T-v))\right]\right\} \mathrm{d} v .
\end{aligned}
$$

Using specific copulas given in terms of a closed analytical expression of $l()$ one can reduce Equation (9). 
Corollary 1. Under assumption of Theorem 4. If the dependence structure is modeled by GumbelBarnett copula then,

$$
\begin{aligned}
K_{\delta}(T) & =\int_{0}^{T} \mathbf{M}_{\Lambda}\left(-v-\frac{e^{-\alpha \delta v}}{\alpha \delta}\right) e^{-\alpha \delta v} \mathrm{~d} v+(1+\theta) \sum_{n=0}^{\infty} \frac{(-T)^{n+1}}{(n+1) !} \\
& \times\left[T \sum_{k=0}^{n+1}\left(\begin{array}{c}
n+1 \\
k
\end{array}\right)(\alpha \delta)^{n+1-k} \mathbf{M}_{\Lambda}^{(k+1)}(0)+(1-n) \sum_{k=0}^{n}\left(\begin{array}{l}
n \\
k
\end{array}\right)(\alpha \delta)^{n-k} \mathbf{M}_{\Lambda}^{(k+1)}(0)\right]
\end{aligned}
$$

The expression of $K_{\delta}(T)$ is similar for the other copulas.

Proof. Using the result in Section 3.2, we have for these copulas.

$$
\begin{aligned}
\Lambda \int_{0}^{T} l(v) e^{-(\alpha \delta+\Lambda) v}(1+(\alpha \delta+\Lambda)(T-v)) \mathrm{d} w & =\Lambda(1+\theta)\left\{\left[2 \frac{1-e^{-(\alpha \delta+\Lambda) T}}{\alpha \delta+\Lambda}\right]\right. \\
& \left.+(\alpha \delta+\Lambda) T \frac{1-e^{-(\alpha \delta+\Lambda) T}}{\alpha \delta+\Lambda}+T e^{-(\alpha \delta+\Lambda) T}\right\} .
\end{aligned}
$$

Moreover

$$
\begin{aligned}
2 \Lambda(1+\theta) \frac{1-e^{-(\alpha \delta+\Lambda) T}}{\alpha \delta+\Lambda} & =2(1+\theta) \sum_{n=0}^{\infty} \frac{(-T)^{n+1}}{(n+1) !} \sum_{k=0}^{n}\left(\begin{array}{c}
n \\
k
\end{array}\right) \Lambda^{k+1}(\alpha \delta)^{n-k} \\
\Lambda(1+\theta)(\alpha \delta+\Lambda) T \frac{1-e^{-(\alpha \delta+\Lambda) T}}{\alpha \delta+\Lambda} & =(1+\theta) T \sum_{n=0}^{\infty} \frac{(-T)^{n+1}}{(n+1) !} \sum_{k=0}^{n+1}\left(\begin{array}{c}
n+1 \\
k
\end{array}\right) \Lambda^{k+1}(\alpha \delta)^{n+1-k} \\
\Lambda(1+\theta) T e^{-(\alpha \delta+\Lambda) T} & =-(1+\theta) \sum_{n=0}^{\infty}(n+1) \frac{(-T)^{n+1}}{(n+1) !} \sum_{k=0}^{n}\left(\begin{array}{l}
n \\
k
\end{array}\right) \Lambda^{k+1}(\alpha \delta)^{n-k} .
\end{aligned}
$$

The result follows by taking the expectation and interchanging the sum and the expectation.

In the next subsection we give a closed-form expression of the constant $K_{\delta}(T)$ where the dependence structure is modeled by some specific well-known copulas.

\subsection{Examples with Some Copulas}

Recall that $e^{x}=\sum_{n=0}^{\infty} \frac{x^{n}}{n !}$ and $(a+b)^{n}=\sum_{k=0}^{n}\left(\begin{array}{l}n \\ k\end{array}\right) a^{k} b^{n-k}$. Let

$$
H=\int_{0}^{T} e^{-\left(\frac{\lambda}{\alpha \delta} e^{-\alpha \delta v}+(\alpha \delta+\lambda) v\right)} \mathrm{d} v
$$


therefore,

$$
\begin{aligned}
H & =\int_{0}^{T} \sum_{n=0}^{\infty} \frac{\left[-\left(\frac{\lambda}{\alpha \delta} e^{-\alpha \delta v}+(\alpha \delta+\lambda) v\right)\right]^{n}}{n !} \mathrm{d} v \\
& =\int_{0}^{T} \sum_{n=0}^{\infty} \frac{(-1)^{n}}{n !}\left(\frac{\lambda}{\alpha \delta} e^{-\alpha \delta v}+(\alpha \delta+\lambda) v\right)^{n} \mathrm{~d} v \\
& =\int_{0}^{T} \sum_{n=0}^{\infty} \frac{(-1)^{n}}{n !} \sum_{k=0}^{n}\left(\begin{array}{l}
n \\
k
\end{array}\right)\left(\frac{\lambda}{\alpha \delta} e^{-\alpha \delta v}\right)^{k}((\alpha \delta+\lambda) v)^{n-k} \mathrm{~d} v \\
& =\int_{0}^{T} \sum_{n=0}^{\infty} \frac{(-1)^{n}}{n !} \sum_{k=0}^{n}\left(\begin{array}{l}
n \\
k
\end{array}\right)(\alpha \delta+\lambda)^{n-k}\left(\frac{\lambda}{\alpha \delta} e^{-\alpha \delta v}\right)^{k} v^{n-k} \mathrm{~d} v \\
& =\sum_{n=0}^{\infty} \frac{(-1)^{n}}{n !} \sum_{k=0}^{n}\left(\begin{array}{l}
n \\
k
\end{array}\right)\left(\frac{\lambda}{\alpha \delta}\right)^{k}(\alpha \delta+\lambda)^{n-k} \int_{0}^{T} e^{-k \alpha \delta v} v^{n-k} \mathrm{~d} v \\
& =\sum_{n=0}^{\infty} \frac{(-1)^{n}}{n !} \sum_{k=0}^{n}\left(\begin{array}{l}
n \\
k
\end{array}\right)\left(\frac{\lambda}{\alpha \delta}\right)^{k}(\alpha \delta+\lambda)^{n-k}(\alpha \delta k)^{k-1-n} \int_{0}^{\alpha \delta k T} e^{-x} x^{n-k} \mathrm{~d} x \\
& =\sum_{n=0}^{\infty} \frac{(-1)^{n}}{n !} \sum_{k=0}^{n}\left(\begin{array}{l}
n \\
k
\end{array}\right)\left(\frac{\lambda}{\alpha \delta}\right)^{k}(\alpha \delta+\lambda)^{n-k}(\alpha \delta k)^{k-1-n} \gamma(n+1-k, \alpha \delta T),
\end{aligned}
$$

where

$$
\gamma(\alpha, x)=\int_{0}^{x} t^{\alpha-1} e^{-t} \mathrm{~d} t \text { is the incomplete gamma function. }
$$

then,

$$
H=\sum_{n=0}^{\infty} \frac{(-1)^{n}}{n !} \sum_{k=0}^{n}\left(\begin{array}{l}
n \\
k
\end{array}\right)\left(\frac{\lambda}{\alpha \delta}\right)^{k}(\alpha \delta+\lambda)^{n-k}(\alpha \delta k)^{k-1-n} \gamma(n+1-k, \alpha \delta T)
$$

Example 1. Ali-Mikhail-Haq copula:

$$
C\left(u_{1,2}\right)=\frac{u_{1} u_{2}}{1-\theta\left(1-u_{1}\right)\left(1-u_{2}\right)}, \theta \in[-1,1) l(v)=1-\theta\left(1-2 e^{-\lambda v}\right) .
$$

If the dependence structure is modeled by the Ali-Mikhail-Haq copula then,

$$
\begin{aligned}
K_{\delta}(T) & =\sum_{n=0}^{\infty} \frac{(-1)^{n}}{n !} \sum_{k=0}^{n}\left(\begin{array}{l}
n \\
k
\end{array}\right)\left(\frac{\lambda}{\alpha \delta}\right)^{k}(\alpha \delta+\lambda)^{n-k}(\alpha \delta k)^{k-1-n} \gamma(n+1-k, \alpha \delta T) \\
& +\lambda T\left(1+\theta-2 \theta \frac{\alpha \delta+\lambda}{\alpha \delta+2 \lambda}\right)+2 \lambda(1+\theta) \bar{a}_{(\alpha \delta+\lambda) T}-\frac{2 \lambda^{2} \theta}{\alpha \delta+2 \lambda} \bar{a}_{(\alpha \delta+2 \lambda) T} .
\end{aligned}
$$

This expression can be easily evaluated numerically.

\section{Proof.}

$$
\begin{aligned}
\lambda \int_{0}^{T} l(v) e^{-(\alpha \delta+\lambda) v}(1+(\alpha \delta+\lambda)(T-v) \mathrm{d} v & =\lambda \int_{0}^{T} l(v) e^{-(\alpha \delta+\lambda) v} \mathrm{~d} v \\
& +\lambda(\alpha \delta+\lambda) \int_{0}^{T} l(v) e^{-(\alpha \delta+\lambda) v}(T-v) \mathrm{d} v,
\end{aligned}
$$

hence,

$$
\begin{aligned}
\lambda \int_{0}^{T} l(v) e^{-(\alpha \delta+\lambda) v} \mathrm{~d} v & =(1+\theta) \lambda \int_{0}^{T} e^{-(\alpha \delta+\lambda) v} \mathrm{~d} v-2 \theta \lambda \int_{0}^{T} e^{-(\alpha \delta+2 \lambda) v} \mathrm{~d} v \\
& =(1+\theta) \lambda\left(\frac{1-e^{-(\alpha \delta+\lambda) T}}{\alpha \delta+\lambda}\right)-2 \lambda \theta\left(\frac{1-e^{(\alpha \delta+2 \lambda) T}}{\alpha \delta+2 \lambda}\right) \\
& =(1+\theta) \lambda \bar{a}_{((\alpha \delta+\lambda) T)}-2 \lambda \theta \bar{a}_{((\alpha \delta+2 \lambda) T)} .
\end{aligned}
$$


Let $x=T-v$ then,

$$
\begin{aligned}
\int_{0}^{T} l(v) e^{-(\alpha \delta+\lambda) T}(T-v) \mathrm{d} v & =\int_{0}^{T} x l(T-x) e^{-(\alpha \delta+\lambda) T-x} \mathrm{~d} v \\
& =e^{-(\alpha \delta+\lambda) T} \int_{0}^{T} x l(T-x) e^{(\alpha \delta+\lambda) x} \mathrm{~d} x \\
& =e^{-(\alpha \delta+\lambda) T} \int_{0}^{T} x\left[1+\theta\left(1-2 e^{-\lambda(T-x)}\right)\right] e^{(\alpha \delta+\lambda) x} \mathrm{~d} x \\
& =e^{-(\alpha \delta+\lambda) T}\left[(1+\theta) \int_{0}^{T} x e^{(\alpha \delta+\lambda) x} \mathrm{~d} x-2 \theta e^{-\lambda T} \int_{0}^{T} x e^{(\alpha \delta+2 \lambda) x} \mathrm{~d} x\right] .
\end{aligned}
$$

Using integration by parts yields

$$
\begin{aligned}
\lambda(\alpha \delta+\lambda) \int_{0}^{T} l(v) e^{-(\alpha \delta+\lambda) T}(T-v) \mathrm{d} v & =\lambda T\left(1+\theta-2 \theta \frac{\alpha \delta+\lambda}{\alpha \delta+2 \lambda}\right) \\
& +\lambda\left((1+\theta) \bar{a}_{(\alpha \delta+\lambda) T}+2 \theta \frac{\alpha \delta+\lambda}{\alpha \delta+2 \lambda} \bar{a}_{(\alpha \delta+2 \lambda) T}\right) .
\end{aligned}
$$

The result follows by combining (11)-(13).

Remark 3. For the FGM copula, $l($.$) is the same as for the Ali-Mikhail-Haq copula; therefore,$ $K_{\delta}(T)$ will be the same.

Example 2. Gumbel-Barnett copula:

$$
C\left(u_{1}, u_{2}\right)=u_{1} u_{2} \exp \left(-\theta \ln u_{1} \ln u_{2}\right), 0 \leq \theta \leq 1, \quad l(v)=1-\theta-\theta \ln \left(1-e^{-\lambda v}\right) .
$$

If the dependence structure is modeled by the Gumbel-Barnett copula then,

$$
\begin{aligned}
K_{\delta}(T) & =\sum_{n=0}^{\infty} \frac{(-1)^{n}}{n !} \sum_{k=0}^{n}\left(\begin{array}{l}
n \\
k
\end{array}\right)\left(\frac{\lambda}{\alpha \delta}\right)^{k}(\alpha \delta+\lambda)^{n-k}(\alpha \delta k)^{k-1-n} \gamma(n+1-k, \alpha \delta T) \\
& +\lambda(1+\theta)\left[(2+(\alpha \delta+\lambda) T) \bar{a}_{(\alpha \delta+\lambda) T}+T e^{-(\alpha \delta+\lambda) T}\right] \\
& +\lambda \theta \sum_{k=2}^{\infty} \frac{1}{k-1}\left[\frac{\alpha \delta+\lambda}{\alpha \delta+\lambda k}\left(T e^{-(\alpha \delta+\lambda k) T}+\bar{a}_{(\alpha \delta+\lambda k) T}\right)+(1+(\alpha \delta+\lambda) T) \bar{a}_{(\alpha \delta+\lambda k) T}\right] .
\end{aligned}
$$

Proof. Let

$$
\kappa(v)=e^{-(\alpha \delta+\lambda) v}(1+(\alpha \delta+\lambda)(T-v)),
$$

then using (14),

$$
\begin{aligned}
\lambda \int_{0}^{T} l(v) \kappa(w) \mathrm{d} v & =\lambda(1+\theta) \int_{0}^{T} e^{-(\alpha \delta+\lambda) v}(1+(\alpha \delta+\lambda)(T-v)) \mathrm{d} v \\
& =\lambda(1+\theta)(1+(\alpha \delta+\lambda) T) \int_{0}^{T} e^{-(\alpha \delta+\lambda) v} \mathrm{~d} v \\
& -\lambda(1+\theta)(\alpha \delta+\lambda) \int_{0}^{T} v e^{-(\alpha \delta+\lambda) v} \mathrm{~d} v
\end{aligned}
$$

using integration by parts yields

$$
\lambda \int_{0}^{T} l(v) \kappa(v) \mathrm{d} v=\lambda(1+\theta)\left[(2+(\alpha \delta+\lambda) T) \bar{a}_{(\alpha \delta+\lambda) T}+T e^{-(\alpha \delta+\lambda) T}\right] .
$$




\section{Moreover,}

$$
\begin{aligned}
\int_{0}^{T} \ln \left(1-e^{-\lambda v}\right) \kappa(v) \mathrm{d} v & =\int_{0}^{T} \sum_{n=1}^{\infty} \frac{e^{-n \lambda v}}{n} e^{-(\alpha \delta+\lambda) v}(1+(\alpha \delta+\lambda)(T-v)) \mathrm{d} v \\
& =\sum_{n=1}^{\infty}\left[(1+(\alpha \delta+\lambda) T) \int_{0}^{T} e^{-(\alpha \delta+(n+1) \lambda) v}\right. \\
& \left.-(\alpha \delta+\lambda) \int_{0}^{T} v e^{-(\alpha \delta+(n+1) \lambda) v}\right] \\
& =\sum_{n=1}^{\infty} \frac{1}{n}\left[(1+(\alpha \delta+\lambda) T) \bar{a}_{(\alpha \delta+(n+1) \lambda) T}\right. \\
& \left.+\frac{\alpha \delta+\lambda}{\alpha \delta+(n+1) \lambda)}\left(T e^{-(\alpha \delta+(n+1) \lambda) T}+\bar{a}_{(\alpha \delta+(n+1) \lambda) T}\right)\right] .
\end{aligned}
$$

By taking $k=n+1$ in the above result and combining it with (11) and (15) yields the result.

\section{Example 3. Marshall-Olkin copula:}

$$
C\left(u_{1}, u_{2}\right)=\min \left(u_{1}^{1-\theta_{1}} u_{2}, u_{1} u_{2}^{1-\theta_{2}}\right) 0 \leq \theta_{1}, \theta_{1} \leq 1 \text { with } l(v)=1-\theta_{1} .
$$

$K_{\delta}(T)$ is given by

$$
\begin{aligned}
K_{\delta}(T) & =\sum_{n=0}^{\infty} \frac{(-1)^{n}}{n !} \sum_{k=0}^{n}\left(\begin{array}{l}
n \\
k
\end{array}\right)\left(\frac{\lambda}{\alpha \delta}\right)^{k}(\alpha \delta+\lambda)^{n-k}(\alpha \delta k)^{k-1-n} \gamma(n+1-k, \alpha \delta T) \\
& +\lambda\left(1+\theta_{1}\right)\left[(2+(\alpha \delta+\lambda) T) \bar{a}_{(\alpha \delta+\lambda) T}+T e^{-(\alpha \delta+\lambda) T}\right] .
\end{aligned}
$$

Example 4. Frank copula:

$$
C\left(u_{1}, u_{2}\right)=-\frac{1}{\theta} \ln \left(1+\frac{\left(e^{-\theta u_{1}}-1\right)\left(e^{-\theta u_{2}}-1\right)}{\left(e^{-\theta}-1\right)}\right), \theta \in \mathbb{R}^{*}, l(v)=\frac{\theta e^{-\theta}}{1-e^{-\theta}} e^{\theta\left(1-e^{-\lambda v}\right)} .
$$

If the dependence is modeled by the Frank copula then,

$$
\begin{aligned}
K_{\delta}(T) & =\sum_{n=0}^{\infty} \frac{(-1)^{n}}{n !} \sum_{k=0}^{n}\left(\begin{array}{l}
n \\
k
\end{array}\right)\left(\frac{\lambda}{\alpha \delta}\right)^{k}(\alpha \delta+\lambda)^{n-k}(\alpha \delta k)^{k-1-n} \gamma(n+1-k, \alpha \delta T) \\
& +\frac{\lambda \theta e^{-\theta}}{1-e^{-\theta}} \sum_{n=0}^{\infty} \frac{\theta^{n}}{n !} \sum_{k=1}^{n+1}\left(\begin{array}{c}
n \\
k-1
\end{array}\right)(-1)^{k-1}\left\{\frac{\alpha \delta+\lambda}{\alpha \delta+\lambda k}\left[\bar{a}_{(\alpha \delta+\lambda k) T}+T e^{-(\alpha \delta+\lambda k) T}\right]\right. \\
& \left.+(1+(\alpha \delta+\lambda) T) \bar{a}_{(\alpha \delta+\lambda k) T}\right\} .
\end{aligned}
$$

Proof. Recall that

$$
l(v)=\frac{\theta e^{-\theta}}{1-e^{-\theta}} e^{\theta\left(1-e^{-\lambda v}\right)}=\frac{\theta e^{-\theta}}{1-e^{-\theta}} \sum_{n=0}^{\infty} \frac{\theta^{n}}{n !} \sum_{j=0}^{n}\left(\begin{array}{c}
n \\
j
\end{array}\right)(-1)^{j} e^{-j \lambda v} .
$$


Then using (14)

$$
\begin{aligned}
\int_{0}^{T} g(v) \kappa(v) \mathrm{d} v & =\frac{\theta e^{-\theta}}{1-e^{-\theta}} \sum_{n=0}^{\infty} \frac{\theta^{n}}{n !} \sum_{j=0}^{n}\left(\begin{array}{c}
n \\
j
\end{array}\right)(-1)^{j}\{(1+(\alpha \delta+\lambda) T) \\
& \left.\times \int_{0}^{T} e^{-(\alpha \delta+(j+1) \lambda) v} \mathrm{~d} v-(\alpha \delta+\lambda) \int_{0}^{T} v e^{-(\alpha \delta+(j+1) \lambda) v} \mathrm{~d} v\right\}, \\
& =\frac{\theta e^{-\theta}}{1-e^{-\theta}} \sum_{n=0}^{\infty} \frac{\theta^{n}}{n !} \sum_{j=0}^{n}\left(\begin{array}{c}
n \\
j
\end{array}\right)(-1)^{j}\left\{(1+(\alpha \delta+\lambda) T) \bar{a}_{(\alpha \delta+(j+1) \lambda) T}\right. \\
& \left.+\frac{\alpha \delta+\lambda}{\alpha \delta+(j+1) \lambda}\left[\bar{a}_{(\alpha \delta+(j+1) \lambda) T}+T e^{-(\alpha \delta+(j+1) \lambda) T}\right]\right\} .
\end{aligned}
$$

By letting $k=j+1$ we have,

$$
\begin{aligned}
\lambda \int_{0}^{T} l(v) \mathcal{K}(v) \mathrm{d} v & =\frac{\lambda \theta e^{-\theta}}{1-e^{-\theta}} \sum_{n=0}^{\infty} \frac{\theta^{n}}{n !} \sum_{k=1}^{n+1}\left(\begin{array}{c}
n \\
k-1
\end{array}\right)(-1)^{k-1}\{(1+(\alpha \delta+\lambda) T) \\
& \left.\times \bar{a}_{(\alpha \delta+k \lambda) T}+\frac{\alpha \delta+\lambda}{\alpha \delta+k \lambda}\left[\bar{a}_{(\alpha \delta+k \lambda) T}+T e^{-(\alpha \delta+k \lambda) T}\right]\right\},
\end{aligned}
$$

The result follows by combining (11) and (16).

\subsection{Non-Homogeneous Poisson Risk Model}

In this subsection, we extend our result by considering a non-homogeneous compound Poisson process where the intensity rate is a deterministic function $\lambda(t)$, which we assume to be piece-wise continuous and bounded on finite intervals.

Theorem 5. Consider a non-homogeneous Poisson compound renewal model with constant force of interest $(\delta>0)$ such that the cumulative distribution function $F \in R V_{-\alpha}$. If Assumption 1 is fulfilled for any time $t \in(0, T]$ then,

$$
\mathbf{P}\left(Z_{\delta}^{n h}(T)>b\right) \sim K_{\delta}(T) \mathbf{P}\left(B_{1}>b\right), \quad b \mapsto \infty
$$

where

$$
K_{\delta}(T)=e^{-m(T)} \sum_{n=1}^{\infty} \int_{0}^{T} \lambda\left(v_{n}\right) \cdots \int_{0}^{v_{2}} \lambda\left(v_{1}\right) \sum_{i=1}^{n} l\left(v_{i}\right) e^{-\alpha \delta \sum_{j=1}^{i} v_{j}} d v_{1} \cdots d v_{n},
$$

and $m(T)=\int_{0}^{T} \lambda(s) \mathrm{d}$ s is mean function (or cumulative intensity function).

Before proving Theorem 5, let us recall the following useful Lemmas.

Lemma 1 (From Ruwet (2007)). Consider a non-homogeneous Poisson process $\mathrm{N}(t)$ with an intensity rate $\lambda(t)$ as a function of $t$. Then the conditional distribution function of the first $n$ occurrence times of the claims, assuming that $\mathrm{N}(t)=n$, has the same distribution as the order statistics of the $n$ i.i.d. uniform random variables $U_{1}, U_{2}, \cdots, U_{n}$ with common distribution

$$
F_{U_{i}}(u)=\frac{m(u)}{m(t)}, 0<u<t, \text { where } m(t)=\int_{0}^{t} \lambda(s) \mathrm{d} s
$$

i.e.,

$$
f_{T_{1}, T_{2}, \cdots, T_{n} \mid \mathrm{N}(t)=n}\left(t_{1}, t_{2}, \cdots, t_{n} \mid n\right)=\frac{n !}{m^{n}(t)} \prod_{i=1}^{n} \lambda\left(t_{i}\right), t_{1}<t_{2}<\cdots<t_{n} .
$$

Lemma 2 (From Asimit and Badescu (2010)). Let $B, X_{1}, X_{2}, \cdots$ be a sequence of independent non-negative random variables with $F$ the cumulative distribution of $B$ such that $F \in \mathcal{S}$. Assume 
further that there exists a constant $M$ such that $\mathbf{P}\left(X_{i}>x\right) \leq \operatorname{MP}(B>b)$ holds for all $b>0$ and any $i=1,2, \cdots$. Then, for any $\epsilon>0$ there exists $\mathrm{A}<\infty$ such that

$$
s_{n}:=\frac{\mathbf{P}\left(\sum_{i=1}^{n} X_{i}>x\right)}{\mathbf{P}(B>b)} \leq \mathrm{A}(1+\epsilon)^{n},
$$

holds for any integer $n$.

Proof of Theorem 5. By conditioning on the number of claims and inter-occurrence time by time $T$, the cumulative distribution functions of $Z_{\delta}(T)$ follow the integral equation

$$
\begin{aligned}
\mathbf{P}\left(Z_{\delta}^{n h}(T)>b\right) & =\sum_{n=1}^{\infty} \mathbf{P}(N(T)=n) \int_{D_{n}} \mathbf{P}\left(\sum_{k=1}^{n} B_{k} e^{-\delta \sum_{i=1}^{k} V_{i}}>b \mid V=v, N(T)\right) \\
& \times \mathbf{P}(V=v \mid N(T)=n) \mathrm{d} v \\
& =\sum_{n=1}^{\infty} \mathbf{P}(N(T)=n) \int_{D_{n}} \mathbf{P}\left(\sum_{k=1}^{n} B_{k} e^{-\delta \sum_{i=1}^{k} V_{i}}>b \mid V=v\right) \\
& \times f_{V_{1}, \cdots, V_{n} \mid N(T)}\left(t_{1}, \cdots t_{n} \mid n\right) \mathrm{d} v .
\end{aligned}
$$

Due to the fact that

$$
\mathbf{P}\left(\sum_{k=1}^{n} B_{k} e^{-\delta \sum_{i=1}^{k} V_{i}}>b \mid V=v\right) \leq \mathbf{P}\left(\sum_{k=1}^{n} B_{k}>b \mid V=v\right),
$$

by the fact that $B_{k} \mid V_{k}=v_{k}$ are i.i.d. and $F \in R V_{-\alpha}$ therefore belongs to $\mathcal{S}$, we can apply Lemma 2. This is true since Assumption 1 implies that there exists a constant $M>0$ such that for all $b>0$ and $v \in(0, T]$, we have

$$
\mathbf{P}\left(B_{1} e^{-\delta V_{1}}>b \mid V_{1}=v_{1}\right) \leq \mathbf{P}\left(B_{1}>b \mid V_{1}=v_{1}\right) \leq M \mathbf{P}\left(B_{1}>b\right) .
$$

From Lemma 2, it follows that for any $\epsilon>0$ there exists $A>0$ such that,

$$
\mathbf{P}\left(\sum_{k=1}^{n} B_{k} e^{-\delta \sum_{i=1}^{k} V_{i}}>b \mid V=v\right) \leq \mathbf{P}\left(\sum_{k=1}^{n} B_{k}>b \mid V=v\right) \leq A(1+\epsilon)^{n} \mathbf{P}\left(B_{1}>b\right)
$$

holds uniformly for all $b>0, v \in(0, T]^{n}$ and $n=1,2, \cdots$.

Then

$$
\begin{gathered}
\sum_{n=1}^{\infty} \mathbf{P}(N(T)=n) \int_{D_{n}} \mathbf{P}\left(\sum_{k=1}^{n} B_{k} e^{-\delta \sum_{i=1}^{k} V_{i}}>b \mid V=v\right) \mathbf{P}(V=v \mid N(T)=n) \\
\leq \\
\sum_{n=1}^{\infty} \mathbf{P}(N(T)=n) \int_{D_{n}} \mathbf{P}\left(\sum_{k=1}^{n} B_{k}>b \mid V=v\right) \mathbf{P}(V=v \mid N(T)=n) \\
\leq \\
\sum_{n=1}^{\infty} \mathbf{P}(N(T)=n) \int_{D_{n}} A(1+\epsilon)^{n} \mathbf{P}\left(B_{1}>b\right) \mathbf{P}(V=v \mid N(T)=n) \\
\leq \\
A \mathbf{P}\left(B_{1}>b\right) \sum_{n=1}^{\infty} \mathbf{P}(N(T)=n)=A \mathbf{P}\left(B_{1}>b\right) P_{N(T)}(1+\epsilon)<\infty .
\end{gathered}
$$

Since $P_{N(T)}$ exists for any $t>0$. 
Using Lemma 1 together with the fact that $\mathbf{P}(N(T)=n)=\frac{m(T)^{n}}{n !} e^{-m(T)}$ we have

$$
\mathbf{P}\left(Z_{\delta}^{n h}(T)>b\right)=\sum_{n=1}^{\infty} e^{-m(T)} \int_{D_{n}} \prod_{i=0}^{n} \lambda\left(v_{i}\right) \mathbf{P}\left(\sum_{k=1}^{n} B_{k} e^{-\delta \sum_{i=1}^{k} V_{i}}>b \mid V=v\right) \mathrm{d} v .
$$

The result follows by applying the Dominated Convergence Theorem in (18).

The following theorem gives the expression of the differential equation of the tail probability of the discounted aggregate claims under a non-homogeneous Poisson risk model.

Theorem 6. Under the assumption of Theorem 5, if the the intensity function $\lambda(t) \neq 0$ for all $t \in[0, T]$, then the deterministic function $K_{\delta}(T)$ given in Theorem 5 satisfied the following differential equation

$$
\frac{\mathrm{d}^{2} K_{\delta}(T)}{\mathrm{d} T^{2}}=\left(\frac{\lambda^{\prime}(T)}{\lambda(T)}+\lambda(T) e^{-\alpha \delta T}-(\lambda(T)+\alpha \delta)\right) \frac{\mathrm{d} K_{\delta}(T)}{\mathrm{d} T}+\lambda(T) l^{\prime}(T) e^{-(m(T)+\alpha \delta T)},
$$

where $K_{\delta}(0)=0,\left.\frac{\partial K_{\delta}(T)}{\partial T}\right|_{T=0}=\lambda(0) l(0)$ and $m(T)=\int_{0}^{T} \lambda(s) \mathrm{d} s$.

Remark 4. If $\lambda(t)=\lambda$ for all $t \in[0, T]$, then $\lambda^{\prime}(t)=0$ and $m(T)=\lambda T$; therefore, the result in Theorem 6 is consistent with the result of Theorem 2

Proof. From Equation (17) of Theorem 5 we have for any $T \geq 0$,

$$
\begin{aligned}
K_{\delta}(T) & =e^{-m(T)} \sum_{n=1}^{\infty} \int_{D_{n}} \prod_{k=1}^{n} \lambda\left(v_{k}\right) \sum_{i=1}^{n} l\left(v_{i}\right) e^{-\alpha \delta \sum_{j=1}^{i} v_{j}} d v \\
& =e^{-m(T)} \sum_{n=1}^{\infty} \int_{0}^{T} \lambda\left(v_{n}\right) \int_{0}^{v_{n}} \lambda\left(v_{n-1}\right) \cdots \int_{0}^{v_{2}} \lambda\left(v_{1}\right) \sum_{i=1}^{n} l\left(v_{i}\right) e^{-\alpha \delta \sum_{j=1}^{i} v_{j}} d v_{1} \cdots d v_{n} \\
& =e^{-m(T)} \sum_{n=1}^{\infty} \int_{0}^{T} \lambda\left(v_{n}\right) \int_{0}^{v_{n}} \lambda\left(v_{n-1}\right) \cdots \int_{0}^{v_{2}} \lambda\left(v_{1}\right) \sum_{i=1}^{n} l\left(v_{i}\right) \prod_{j=1}^{i} e^{-\alpha \delta v_{j}} d v_{1} \cdots d v_{n} \\
& =e^{-m(T)}\left\{\int_{0}^{T} \lambda(v) l(v) e^{-\alpha \delta v} d v+\sum_{n=2}^{\infty} \int_{0}^{T} \int_{0}^{v_{n}} \lambda\left(v_{n-1}\right)\right. \\
& \left.\cdots \int_{0}^{v_{2}} \lambda\left(v_{1}\right) \sum_{i=1}^{n} l\left(v_{i}\right) e^{-\alpha \delta \sum_{j=1}^{i} v_{j}} d v_{1} \cdots d v_{n}\right\} .
\end{aligned}
$$


As in Theorem 2, the derivative of the preceding expression with respect to $T$ yields.

$$
\begin{aligned}
\frac{\mathrm{d} K_{\delta}(T)}{\mathrm{d} T}= & -m^{\prime}(T) K_{\delta}(T)+e^{-m(T)} \lambda(T) l(T) e^{-\alpha \delta T}+e^{-m(T)} \lambda(T) \sum_{n=2}^{\infty} \int_{0}^{T} \lambda\left(v_{n-1}\right) \\
& \ldots \int_{0}^{v_{2}} \lambda\left(v_{1}\right)\left[\sum_{i=1}^{n-1} l(v) \prod_{j=1}^{i} e^{-\alpha \delta v_{j}}+l(v) e^{-\alpha \delta \sum_{j=1}^{n-1} v_{j}} e^{-\alpha \delta T}\right] d v_{1} \cdots d v_{n-1} \\
& =-\lambda(T) K_{\delta}(T)+\lambda(T) l(T) e^{-(m(T)+\alpha \delta T)}+e^{-m(T)} \lambda(T)\{ \\
& \sum_{n=2}^{\infty} \int_{0}^{T} \lambda\left(v_{n-1}\right) \cdots \int_{0}^{v_{2}} \lambda\left(v_{1}\right) \sum_{i=1}^{n-1} l(v) \prod_{j=1}^{i} e^{-\alpha \delta v_{j}} d v_{1} \cdots d v_{n-1} \\
& \left.+e^{-\alpha \delta T} \sum_{n=2}^{\infty} \int_{0}^{T} \lambda\left(v_{n-1}\right) \cdots \int_{0}^{v_{2}} \lambda\left(v_{1}\right) l(v) e^{-\alpha \delta \sum_{j=1}^{n-1} v_{j}} d v_{1} \cdots d v_{n-1}\right\} \\
= & -\lambda(T) K_{\delta}(T)+\lambda(T) l(T) e^{-(m(T)+\alpha \delta T)} \\
& +e^{-m(T)} \lambda(T) \sum_{n=1}^{\infty} \int_{0}^{T} \lambda\left(v_{n}\right) \cdots \int_{0}^{v_{2}} \lambda\left(v_{1}\right) \sum_{i=1}^{n} l(v) \prod_{j=1}^{i} e^{-\alpha \delta v_{j}} d v_{1} \cdots d v_{n} \\
& +e^{-(m(T)+\alpha \delta T)} \lambda(T) \sum_{n=1}^{\infty} \int_{0}^{T} \lambda\left(v_{n}\right) \cdots \int_{0}^{v_{2}} \lambda\left(v_{1}\right) l(v) e^{-\alpha \delta \sum_{j=1}^{n} v_{j}} d v_{1} \cdots d v_{n} \\
= & -\lambda(T) K_{\delta}(T)+\lambda(T) l(T) e^{-(m(T)+\alpha \delta T)}+\lambda(T) K_{\delta}\left(T+e^{-(m(T)+\alpha \delta T)} \lambda(T)\right) \\
& \times \sum_{n=1}^{\infty} \int_{0}^{T} \lambda\left(v_{n}\right) \int_{0}^{v_{n}} \lambda\left(v_{n-1}\right) \cdots \int_{0}^{v_{2}} \lambda\left(v_{1}\right) l(v) e^{-\alpha \delta \sum_{j=1}^{n} v_{j}} d v_{1} \cdots d v_{n} \\
= & \lambda(T) e^{-(m(T)+\alpha \delta T)} l(T)+ \\
+ & \lambda(T) e^{-(m(T)+\alpha \delta T)} \sum_{n=1}^{\infty} \int_{0}^{T} \lambda\left(v_{n}\right) \cdots \int_{0}^{v_{2}} \lambda\left(v_{1}\right) l(v) e^{-\alpha \delta \sum_{j=1}^{n} v_{j}} d v_{1} \cdots d v_{n} .
\end{aligned}
$$

By taking the derivative of the preceding expression with respect to $T$ we have,

$$
\begin{aligned}
\frac{\mathrm{d}^{2} K_{\delta}(T)}{\mathrm{d} T^{2}} & =\left(\frac{\lambda^{\prime}(T)}{\lambda(T)}-(\alpha \delta+\lambda(T))\right) \frac{\mathrm{d} K_{\delta}(T)}{\mathrm{d} T}+\lambda(T) e^{-(m(T)+\alpha \delta T)}\left\{l^{\prime}(T)+\lambda(T) l(T) e^{-\alpha \delta T}\right. \\
& \left.+\lambda(T) \sum_{n=2}^{\infty} \int_{0}^{T} \lambda\left(v_{n-1}\right) \cdots \int_{0}^{v_{2}} \lambda\left(v_{1}\right) l(v) e^{-\alpha \delta \sum_{j=1}^{n-1} v_{j}} e^{-\alpha \delta T} d v_{1} \cdots d v_{n-1}\right\} \\
& =\left(\frac{\lambda^{\prime}(T)}{\lambda(T)}-(\alpha \delta+\lambda(T))\right) \frac{\mathrm{d} K_{\delta}(T)}{\mathrm{d} T}+\lambda(T) e^{-(m(T)+\alpha \delta T)}\left\{l^{\prime}(T)+\lambda(T) l(T) e^{-\alpha \delta T}\right. \\
& \left.+\lambda(T) e^{-\alpha \delta T} \sum_{n=1}^{\infty} \int_{0}^{T} \lambda\left(v_{n}\right) \cdots \int_{0}^{v_{2}} \lambda\left(v_{1}\right) l(v) e^{-\alpha \delta \sum_{j=1}^{n} v_{j}} d v_{1} \cdots d v_{n}\right\} .
\end{aligned}
$$

From Equation (20) we have,

$$
\lambda(T) e^{-\alpha \delta T} \sum_{n=1}^{\infty} \int_{0}^{T} \lambda\left(v_{n}\right) \cdots \int_{0}^{v_{2}} \lambda\left(v_{1}\right) l(v) e^{-\alpha \delta \sum_{j=1}^{n} v_{j}} \prod_{i=1}^{n} \mathrm{~d} v_{i}=e^{m(T)} \frac{\mathrm{d} K_{\delta}(T)}{\mathrm{d} T}-\lambda(T) l(T) e^{-\alpha \delta T} .
$$

Then, substituting (22) into (21) leads to,

$$
\begin{aligned}
\frac{\mathrm{d}^{2} K_{\delta}(T)}{\mathrm{d} T^{2}} & =\left(\frac{\lambda^{\prime}(T)}{\lambda(T)}-(\alpha \delta+\lambda(T))\right) \frac{\mathrm{d} K_{\delta}(T)}{\mathrm{d} T}+\lambda(T) e^{-(m(T)+\alpha \delta T)}\left\{l^{\prime}(T)+\lambda(T) l(T) e^{-\alpha \delta T}\right. \\
& \left.+e^{m(T)} \frac{\mathrm{d} K_{\delta}(T)}{\mathrm{d} T}-\lambda(T) l(T) e^{-\alpha \delta T}\right\} \\
& =\left(\frac{\lambda^{\prime}(T)}{\lambda(T)}-(\alpha \delta+\lambda(T))\right) \frac{\mathrm{d} K_{\delta}(T)}{\mathrm{d} T}+\lambda(T) e^{-(m(T)+\alpha \delta T)}\left\{l^{\prime}(T)+e^{m(T)} \frac{\mathrm{d} K_{\delta}(T)}{\mathrm{d} T}\right\} \\
& =\left(\frac{\lambda^{\prime}(T)}{\lambda(T)}+\lambda(T) e^{-\alpha \delta T}-(\alpha \delta+\lambda(T))\right) \frac{\mathrm{d} K_{\delta}(T)}{\mathrm{d} T}+\lambda(T) l^{\prime}(T) e^{-(m(T)+\alpha \delta T)} .
\end{aligned}
$$


The following theorem gives the solution of the differential equation of $K_{\delta}(T)$ where the intensity function is not a constant.

Theorem 7. Under the assumption of Theorem 6. If we assume further that $K_{\delta}^{\prime}(t)>0$ for every $t \in[0, T]$, then

$$
K_{\delta}(T)=\int_{0}^{T} \lambda(v) e^{-(m(v)+\alpha \delta v)} e^{\int_{0}^{v} \lambda(s) e^{-\alpha \delta s} \mathrm{~d} s} \mathrm{~d} v+\int_{0}^{T} \int_{0}^{v} \lambda(s) l^{\prime}(s) e^{-(m(s)+\alpha \delta s)} \mathrm{d} s \mathrm{~d} v .
$$

\section{Proof.}

(1) Firstly let us solve Equation (19) of Theorem 6 without the second member.

$$
\text { From } \frac{\mathrm{d}^{2} K_{\delta}(T)}{\mathrm{d} T^{2}}=\left(\frac{\lambda^{\prime}(T)}{\lambda(T)}+\lambda(T) e^{-\alpha \delta T}-\alpha \delta-\lambda(T)\right) \frac{\mathrm{d} K_{\delta}(T)}{\mathrm{d} T}
$$

we have,

$$
\begin{aligned}
\frac{\mathrm{d}}{\mathrm{d} T}\left(\ln \left[\frac{\mathrm{d} L_{\delta}(T)}{\mathrm{d} T}\right]\right) & =\frac{\lambda^{\prime}(T)}{\lambda(T)}+\lambda(T) e^{-\alpha \delta T}-\alpha \delta-\lambda(T) \\
\ln \left[\frac{\mathrm{d} L_{\delta}(T)}{\mathrm{d} T}\right] & =\ln \lambda(T)-(m(T)+\alpha \delta T)+\int_{0}^{T} \lambda(s) e^{-\alpha \delta s} \mathrm{~d} s+\mu_{0} \\
\frac{\mathrm{d} L_{\delta}(T)}{\mathrm{d} T} & =\lambda(T) e^{-(m(T)+\alpha \delta T)} e^{\int_{0}^{T} \lambda(s) e^{-\alpha \delta s} \mathrm{~d} s}+\mu_{1} \\
L_{\delta}(T) & =\int_{0}^{T} \lambda(v) e^{-(m(v)+\alpha \delta v)} e^{\int_{0}^{v} \lambda(s) e^{-\alpha \delta s} \mathrm{~d} s} \mathrm{~d} v+\mu .
\end{aligned}
$$

By letting $\mu=0$ we have,

$$
L_{\delta}(T)=\int_{0}^{T} \lambda(v) e^{-(m(v)+\alpha \delta v)} e^{\int_{0}^{v} \lambda(s) e^{-\alpha \delta s} \mathrm{~d} s} \mathrm{~d} v
$$

(2) Secondly let us find the solution of Equation (19) of Theorem (6) for the second member.

Let for any $T \geq 0$,

$$
H(T)=\int_{0}^{T} \int_{0}^{v} \lambda(s) l^{\prime}(s) e^{-(m(s)+\alpha \delta s)} \mathrm{d} s \mathrm{~d} v .
$$

One can easily check that

$$
\frac{\mathrm{d}^{2} H(T)}{\mathrm{d} T^{2}}=\lambda(T) l^{\prime}(T) e^{-(m(T)+\alpha \delta T)} .
$$

Therefore, the final solution is

$$
\mathbf{K}_{\delta}(T)=L_{\delta}(T)+H(T),
$$

which proves the statement.

\section{Applications}

In this section we use the expression of $K_{\delta}(T)$ to derive an upper bound of the ruin probability and also to evaluate some risk measure such as the value-at-risk.

\subsection{Ruin Probability}

Finding the exact analytical expression of the ruin probability that can be computationally tractable is an extremely complex task. For simple cases of the collective risk model, exact formulas and approximations have been found for the ruin probability. However, 
when the effect of the force of interest is incorporated into the collective risk model, the calculations become more difficult. In the case of the discounted compound Poisson risk model, with a constant force of interest, a differential equation has been obtained for the ruin probability and a solution has been found when the amount of the claims follows an exponential distribution. For more details, see Sundt and Teugels (1995). The calculation of the ruin probability is also possible if we know the distribution of our risk process. The expressions obtained by Leveillé and Hamel (2018); Léveillé et al. (2010) for the distribution of the discounted aggregated renewal sum confirm that it will be very difficult to find an explicit expression of the probability of ruin in the framework defined by the previous authors. Hence the use of bounds for an estimation of the probability of ruin. Several bounds have been proposed in the framework of Sparre Andersen's model, see Cai and Dickson (2003), when the force of interest is constant and Cai (2002) when the force of interest follows a time series. Other authors such as Albrecher and Teugels (2006), Tang (2005) and Wei and Yang (2004) have incorporated the dependency into the risk model and have derived asymptotic formulas for finite and infinite time ruin probabilities. In this subsection, we present some asymptotic results of the ruin probability for homogeneous and non-homogeneous compound Poisson risk model with a heavy tailed distribution for the claim severities and we also assume that the dependence structure is given in Section 3.2. This subsection provides numerical illustration with the use of copulas.

Let

$$
U_{\delta}(t)=b e^{\delta t}+C_{\delta}(t)-e^{\delta t} Z_{\delta}(t)
$$

where $b$ is the surplus at time $t=0$ and $C_{\delta}(t)=\int_{0}^{t} e^{\delta(t-s)} \mathrm{d} C(s)$ represents the accumulated amount of premiums received at time $t$. The non-decreasing and right continuous stochastic process, $\{C(s)\}_{s \geq 0}$ with $C(0)=0$ represents the total amount of premiums accumulated to time $s$. We then define the time to ruin as

$$
T_{\delta, b}=\inf \left\{t>0: U_{\delta}(t)<0 \mid U_{\delta}(0)=b\right\},
$$

therefore, the finite-time ruin probability is given by

$$
\psi_{\delta}(b, T)=\mathbf{P}\left(T_{\delta, b} \leq T\right) .
$$

Clearly

$$
\mathbf{P}\left(Z_{\delta}(T) \geq b+e^{-\delta T} C_{\delta}(T)\right) \leq \psi_{\delta}(b, T) \leq \mathbf{P}\left(Z_{\delta}(T) \geq b\right), \text { holds for } \delta \geq 0 .
$$

Using the long-tailed property of subexponential distributions, see Embrechts et al. (1997), in (26) leads to the following Corollary of Theorems 1 and 5.

Corollary 2. Consider a homogeneous and a non-homogeneous compound renewal risk process with constant force of interest $\delta>0$ such that Assumption 1 is fulfilled for any $t \in(0 ; T]$, assume further that $F \in R V_{-\alpha}$. In addition, if $C_{\delta}(T)<\infty$ then

$$
\psi_{\delta}(b, T) \sim K_{\delta}(T) \mathbf{P}\left(B_{1} \geq b\right), \quad b \rightarrow+\infty .
$$

Proof. See Asimit and Badescu (2010).

Numerical Illustration

In this subsection, we provide numerical illustrations for the asymptotic finite-time ruin probability. For this purpose we assume that the counting process is a homogeneous, a non-homogeneous and a mixed Poisson process. Furthermore, we assume that the dependence structure is given by some copulas defined in Section 3.2. However, the 
forthcoming claim amounts are assumed to have a Weibull distribution with cumulative distribution given by:

$$
F_{B_{1}}(b)=1-\exp \left(-b^{\frac{1}{\tau}}\right)
$$

Insurance claims are known to be right-skewed. Moreover, under extreme events scenarios the frequency of the claim occurrence is relatively low; therefore, we choose our parameters accordingly.

In Table 1, we consider a homogeneous compound Poisson process where $\lambda=0.5$, the constant force of interest rate is assumed to be $\delta=20 \%, \tau=2$ and the time horizon $T \in\{20,40\}$.

In Table 2, we consider a mixed homogeneous compound Poisson process where $\Lambda$ has gamma distribution with density

$$
f_{\Lambda}(\lambda)=\frac{\beta^{a}}{\Gamma(a)} \lambda^{a-1} \exp (-\beta \lambda), \text { for } \lambda \geq 0,
$$

where $a=15, \beta=20$.

In Table 3, we consider a non-homogeneous Poisson risk model where the intensity function is assumed to be

$$
\lambda(t)=0.5+0.2 \exp (-0.2 t)
$$

and the other parameters were chosen as in Table 1.

Tables 1-3 give numerical values for the ruin probability respectively for the AliMikhail-Haq and Frank copulas.

Table 1. Asymptotic finite-time ruin probability for homogeneous Poisson risk model with interest rate.

\begin{tabular}{ccccccccc}
\hline$\alpha$ & \multicolumn{9}{c}{$\alpha=2.5$} \\
\hline$\theta$ & \multicolumn{9}{c}{$\theta=-0.5$} & \multicolumn{2}{c}{$\theta=0.75$} \\
\hline$T$ & \multicolumn{2}{c}{$T=20$} & \multicolumn{2}{c}{$T=40$} & \multicolumn{2}{c}{$T=20$} & \multicolumn{2}{c}{$T=40$} \\
\hline$b$ & 50 & 100 & 50 & 100 & 50 & 100 & 50 & 100 \\
\hline$\psi_{\delta}^{\text {Ali }}(b, T)$ & 0.0074 & 0.0004 & 0.0145 & 0.0008 & 0.0112 & 0.0006 & 0.0218 & 0.0012 \\
\hline$\psi_{\delta}^{\text {Frank }}(b, T)$ & 0.0185 & 0.0010 & 0.0365 & 0.0020 & 0.0171 & 0.0009 & 0.0339 & 0.0018 \\
\hline
\end{tabular}

Table 2. Asymptotic ruin probability for mixed homogeneous Poisson risk model with interest rate.

\begin{tabular}{ccccccccc}
\hline$\alpha$ & \multicolumn{9}{c}{$\alpha=2.5$} \\
\hline$\theta$ & \multicolumn{9}{c}{$\theta=-0.5$} & \multicolumn{2}{c}{$\theta=0.75$} \\
\hline$T$ & \multicolumn{2}{c}{$T=20$} & \multicolumn{2}{c}{$T=40$} & \multicolumn{2}{c}{$T=20$} & \multicolumn{2}{c}{$T=40$} \\
\hline$b$ & 50 & 100 & 50 & 100 & 50 & 100 & 50 & 100 \\
\hline$\psi_{\delta}^{\text {Ali }}(b, T)$ & 0.0113 & 0.0006 & 0.0225 & 0.0012 & 0.0155 & 0.0008 & 0.0306 & 0.0016 \\
\hline$\psi_{\delta}^{\text {Frank }}(b, T)$ & 0.0271 & 0.0015 & 0.0540 & 0.0029 & 0.0259 & 0.0014 & 0.0517 & 0.0028 \\
\hline
\end{tabular}


Table 3. Asymptotic ruin probability for non-homogeneous Poisson risk model with interest rate.

\begin{tabular}{|c|c|c|c|c|c|c|c|c|}
\hline$\theta$ & \multicolumn{8}{|c|}{$\theta=-0.5$} \\
\hline$\alpha$ & \multicolumn{4}{|c|}{$\alpha=2.5$} & \multicolumn{4}{|c|}{$\alpha=5$} \\
\hline$T$ & \multicolumn{2}{|c|}{$T=20$} & \multicolumn{2}{|c|}{$T=40$} & \multicolumn{2}{|c|}{$T=20$} & \multicolumn{2}{|c|}{$T=40$} \\
\hline$b$ & 50 & 100 & 50 & 100 & 50 & 100 & 50 & 100 \\
\hline$\psi_{\delta}^{A l i}(b, T)$ & 0.0310 & 0.0017 & 0.0622 & 0.0033 & 0.0249 & 0.0013 & 0.0498 & 0.0027 \\
\hline$\psi_{\delta}^{\text {Frank }}(b, T)$ & 0.0365 & 0.0019 & 0.0743 & 0.0040 & 0.0365 & 0.0020 & 0.0744 & 0.0040 \\
\hline
\end{tabular}

Our numerical illustrations show (see Tables 1 and 2) that when the time to maturity increases, the ruin probability for the homogeneous and mixed Poisson processes increases. However, the inverse effect is observed for an increase of the copula's parameter for the Frank copula. For non-homogeneous risk model (see Table 3), increasing the parameter $\alpha$ decreases the ruin probability in the case of the Ali-Mikhail-Haq copula.

\subsection{Risk Management}

In the movement to redefine the solvency margin according to risks for all financial risks, after the bank industry and the implementation of the Basel II agreement, it was the turn of insurance industry to see its regulations adapted to integrate risk. After Solvency I, which provided for a solvency margin determined as a function of percentages on premiums and claims, insurance regulations moved to more complex rules integrating risk, either by applying a standard formula or by using an internal model. Note that the subprime crisis, far from having put this reform on hold, convinced the European Commission to legislate to protect itself against so-called systemic risks (chain failures of players-banks, insurers, etc.-in the financial world).

The second pillar aims to set qualitative standards for monitoring risks internally in companies and how the supervisory authority should exercise its supervisory powers in this context. The identification of the "riskiest" companies is an objective, and the supervisory authorities will have in their power the possibility of requiring these companies to hold a capital higher than the amount suggested by the calculation of the so-called solvency capital, requirement (SCR) and/or reduce their exposure to risks. See Hoiring (2013), Gatzert and Wesker (2012) and Boonen (2017). This capital requirement covers all the risks that an insurer faces and is defined as the value-at-risk (VaR) of the basic own funds subject to a confidence level of $99.5 \%$ on a 1-year period. In this subsection we provide an asymptotic expression for the value-at-risk.

\subsubsection{Value-at-Risk}

Considered as the most popular risk measure in financial industry, the value-at-risk $(\mathrm{VaR})$ at level $p$ for a loss variable $L$ represents the $p$-quantile, defined as in Jorion (2001):

$$
\operatorname{VaR}_{p}(L)=\inf \{b \in \mathbb{R}: \mathbf{P}(L>b) \leq 1-p\} .
$$

An alternative definition of the value-at-risk $(\mathrm{VaR})$ is the $\alpha$-quantile of the distribution of the discounted aggregate sum of claims. Using Theorems 1 and 5, this quantile can be expressed as:

$$
\operatorname{VaR}_{1-p}\left(Z_{\delta}(T)\right) \sim \operatorname{VaR}_{1-\frac{p}{K_{\delta}}}\left(B_{1}\right), \text { for } p \downarrow 0,
$$

provided that the density function of $Z_{\delta}(T)$ is continuously close enough to the right tail.

\subsubsection{Numerical Illustration}

In this subsection, we provide numerical illustrations for the value-at-risk where Weibull distribution is considered for the claim severities using some copulas defined in 
Section 3.2. With the same parameters as before, we compute the value-at-risk at level $p=0.5 \%$.

In Tables 4 and 5, we note that the value-at-risk is higher in the case of a negative value of $\theta$ for the Frank copula and the inverse effect is observed for the Ali-Mikhail-Haq copula. For the non-homogeneous Poisson process (see Table 6), the value-at risk is positively correlated to the parameter $\alpha$ in the case of the Frank copula and negatively correlated to the parameter $\alpha$ for the Ali-Mikhail-Haq copula.

Table 4. Asymptotic value-at-risk for homogeneous Poisson risk model with interest rate.

\begin{tabular}{|c|c|c|c|c|c|c|c|}
\hline \multirow{2}{*}{\multicolumn{2}{|c|}{$\frac{\theta}{\text { Time horizon }}$}} & \multicolumn{3}{|c|}{$\theta=-0.5$} & \multicolumn{3}{|c|}{$\theta=0.75$} \\
\hline & & $T=10$ & $T=20$ & $T=40$ & $T=10$ & $T=20$ & $T=40$ \\
\hline Ali & $\mathrm{VaR}_{99.5 \%}$ & 46.5245 & 55.7628 & 66.20207 & 52.3648 & 62.0655 & 73.0105 \\
\hline Frank & $\mathrm{VaR}_{99.5 \%}$ & 59.4932 & 70.2307 & 82.0888 & 58.2135 & 68.9356 & 80.7418 \\
\hline
\end{tabular}

Table 5. Asymptotic value-at-risk for mixed homogeneous Poisson risk model with interest rate.

\begin{tabular}{|c|c|c|c|c|c|c|c|}
\hline \multicolumn{2}{|c|}{$\theta$} & \multicolumn{3}{|c|}{$\theta=-0.5$} & \multicolumn{3}{|c|}{$\theta=0.75$} \\
\hline & rizon & $T=10$ & $T=20$ & $T=40$ & $T=10$ & $T=20$ & $T=40$ \\
\hline Ali & $\mathrm{VaR}_{99.5 \%}$ & 51.9694 & 62.2281 & 73.5260 & 56.8626 & 67.3297 & 78.9272 \\
\hline Frank & $\operatorname{VaR}_{99.5 \%}$ & 65.3088 & 76.7875 & 89.3044 & 64.4641 & 75.9769 & 88.4878 \\
\hline
\end{tabular}

Table 6. Asymptotic value-at-risk for non-homogeneous Poisson risk model with interest rate.

\begin{tabular}{|c|c|c|c|c|c|c|c|}
\hline \multirow{2}{*}{\multicolumn{2}{|c|}{$\begin{array}{c}\alpha \\
\text { Time horizon }\end{array}$}} & \multicolumn{3}{|c|}{$\alpha=2.5$} & \multicolumn{3}{|c|}{$\alpha=5$} \\
\hline & & $T=10$ & $T=20$ & $T=40$ & $T=10$ & $T=20$ & $T=40$ \\
\hline Ali & $\mathrm{VaR}_{99.5 \%}$ & 67.1523 & 79.1319 & 92.0187 & 63.8356 & 75.3102 & 87.7768 \\
\hline Frank & $\mathrm{VaR}_{99.5 \%}$ & 69.3052 & 82.0407 & 95.4547 & 69.3582 & 82.0685 & 95.4694 \\
\hline
\end{tabular}

\section{Discussion and Conclusions}

Under the model assumption, the numerical simulations show that the ruin is high for the Frank copula in comparison with the Ali-Mikhail-Haq copula in both homogeneous, mixed homogeneous and non-homogeneous Poisson processes. Moreover, the ruin is higher when the claim number process is modeled by a non-homogeneous process than the other process understudies. This finding was further confirmed by the simulation of the value-at-risk as shown in Table 6. One of the most important advantages of simulation in social science is the fact that simulation allows to approach the conditions of the experiment. It is very generally accepted that only experimentation can ensure the existence of causeand-effect. Indeed, it is by manipulating the cause under experimental conditions (i.e., controlling other variables) that one can ensure a variation of the effect. Another major advantage of the simulation approach is that it helps with the discovery. Therefore, although the findings in this study are interesting and important from a theoretical point of view, further research must be done in terms of empirical studies with real data to confirm our findings.

Author Contributions: This doctoral research paper was done by K.E. under the supervision of F.A. Both authors have read and agreed to the published version of the manuscript.

Funding: This research was funded by the Global Excellence and Stature (GES) 4.0 scholarship of the University of Johannesburg (UJ). 
Acknowledgments: The authors would like to thank the editor and four anonymous referees for many constructive comments which greatly helped to improve the quality of this paper.

Conflicts of Interest: The authors declare no conflict of interest.

\section{References}

Albrecher, Hansjörg, and Jef L. Teugels. 2006. Exponential Behavior in the Presence of Dependence in Risk Theory. Journal of Applied Probability 43: 257-73. [CrossRef]

Albrecher, Hansjörg, and Onno J. Boxma. 2004. A ruin model with dependence between claim sizes and claim intervals. Insurance: Mathematics and Economics 35: 245-54. [CrossRef]

Albrecher, Hansjörg, and Onno J. Boxma. 2005. On the discounted penalty function in a Markov dependent risk model. Insurance: Mathematics and Economics 37: 650-72. [CrossRef]

Asimit, Alexandru V., and Andrei L. Badescu. 2010. Extremes on the discounted aggregate claims in a time dependent risk model. Scandinavian Actuarial Journal 93-104. [CrossRef]

Boogaert, P., J. Haezendonck, and F. Delbaen. 1988. Limit theorems for the present value of the surplus of an insurance portfolio. Insurance: Mathematics and Economics 72: 131-38. [CrossRef]

Boonen, Tim J. 2017. Solvency II solvency capital requirement for life insurance companies based on expected shortfall. European Actuarial Journal. [CrossRef]

Boudreault, Mathieu, Helene Cossette, David Landriault, and Etienne Marceau. 2006. On a Risk Model with Dependence between inter-claim Arrivals and Claim Sizes. Scandinavian Actuarial Journal 5: 265-85. [CrossRef]

Cai, Jun. 2002. Ruin probabilities with dependent rates of interest. Journal of Applied Probability 39: 312-23. [CrossRef]

Cai, Jun, and David Dickson. 2003. Upper bounds for ultimate ruin probabilities in the Sparre Andersen model with interest. In Insurance: Mathematics and Economics. Amsterdam: Elsevier, vol. 32, pp. 61-71.

Delbaen, Freddy, and Jean Haezendonck. 1987. Classical risk theory in an economic environment. Insurance: Mathematics and Economics 6: 85-116. [CrossRef]

Embrechts, Paul, Claudia Klüppelberg, and Thomas Mikosch. 1997. Modelling of Extremal Events for Insurance and Finance. Berlin: Springer.

Gatzert, Nadine, and Hannah Wesker. 2012. A Comparative Assessment of Basel II/III and Solvency II. The Geneva Papers 37: 539-70. [CrossRef]

Geluk, Jaap, and Qihe Tang. 2009. Asymptotic Tail Probabilities of Sums of Dependent Subexponential Random Variables. Journal of Theoretical Probability 22: 871-82. [CrossRef]

Hoiring, Dirk. 2013. Will Solvency II Market Risk Requirements Bite? The Impact of Solvency II on Insurers' Asset Allocation. The Geneva Papers on Risk and Insurance Issues and Practice 38: 250-73. [CrossRef]

Jorion, Philippe. 2001. Value at Risk, 2nd ed. New York: McGraw-Hill.

Léveillé, Ghislain, and Emmanuel Hamel. 2018. Conditional, Non-Homogeneous and Doubly Stochastic Compound Poisson Processes with Stochastic Discounted Claims. Methodology and Computing in Applied Probability. [CrossRef]

Léveillé, Ghislain, and Franck Adékambi. 2011. Covariance of discounted compound renewal sums with a stochastic interest rate. Scandinavian Actuarial Journal 2: 138-53. [CrossRef]

Leveillé, Ghislain, and Franck Adékambi. 2012. Joint moments of discounted compound renewal sums. Scandinavian Actuarial Journal 1: 40-55. [CrossRef]

Léveillé, Ghislain, and José Garrido. 2001a. Moments of compound renewal sums wit discounted claims. Insurance: Mathematics and Economics 28: 217-31.

Léveillé, Ghislain, and José Garrido. 2001b. Recursive moments of compound renewal sums with discounted claims. Scandinavian Actuarial Journal 2: 98-110. [CrossRef]

Léveillé, Ghislain, José Garrido, and Ya Fang Wang. 2010. Moment generating functions of compound renewal sums with discounted claims. Scandinavian Actuarial Journal 3: 165-84. [CrossRef]

Liu, Xijun, and Qingwu Gao. 2015. Uniformly asymptotic behavior for the tail probability of discounted aggregate claims in the time-dependent risk model with upper tail asymptotically independent claims. Communications in Statistics Theory and Methods. [CrossRef]

Lu, Dawei, Lixin Song, and Fuqi Li. 2018. Uniform asymptotics for discounted aggregate claims in dependent multi-risk model. In Communications in Statistics-Theory and Methods. Oxford: Taylor \& Francis, vol. 48, pp. 781-93.

Nelsen, Roger B. 1999. An Introduction to Copulas. New York: Springer.

Ruwet, Christel. 2007. Processus de Poisson. Belgique: Université de Liège.

Sklar, A. 1959. Fonctions de Répartition à n Dimensions et Leurs Marges. Paris: Publications de l'Institut de Statistique de l' Université de Paris, vol. 8, pp. 229-31.

$\mathrm{Su}, \mathrm{Chun}$, Zhishui $\mathrm{Hu}, \mathrm{Yu}$ Chen, and Hanying Liang. 2007. A wide class of heavy-tailed distributions and its applications. Frontiers of Mathematics in China 2: 257-86. [CrossRef]

Sundt, Bjørn, and Jozef L. Teugels. 1995. Ruin estimate under interest force. Insurance: Mathematics and Economics 16: 7-22. [CrossRef]

Tang, Qithe. 2005. The Finite-time Ruin Probability of the Compound Poisson Model with Constant Interest Force. Journal of Applied Probability 42: 608-19. [CrossRef] 
Taylor, Gregory Clive. 1979. Probability of ruin under inflationary conditions or under experience rating. Astin Bulletin 10: 149-62. [CrossRef]

Waters, Howard R. 1983. Probability of ruin for a risk process with claims cost inflation. Scandinavian Actuarial Journal 3: 148-64. [CrossRef]

Wei, Li, and Hai-liang Yang. 2004. Explicit Expressions for the Ruin Probabilities of Erlang Risk Processes with Pareto Individual Claim. Acta Mathematicae Applicatae Sinica, English Series 20: 495-506. [CrossRef]

Willmot, Gordon E. 1989. The total claims distribution under inflationary conditions. Scandinavian Actuarial Journal 10: 1-12. [CrossRef]

Yang, Yang, Kaiyong Wang, and Dimitrios G. Konstantinides. 2014. Uniform asymptotics for discounted aggregate claims in dependent risk models. Journal of Applied Probability 51: 669-84. [CrossRef]

Yang, Yang, Remigijus Leipus, and Jonas Šiaulys. 2012. Tail probability of randomly weighted sums of subexponential random variables under dependence structure. Statistics and Probability Letters 82: 1727-36. [CrossRef]

Yuen, Kam C., Guojing Wang, and Rong Wu. 2006. On the renewal risk process with stochastic interest. Stochastic Processes and their Applications 116: 1496-510. [CrossRef]

Zhang, Zhi-min, and Hu Yang. 2011. The compound Poisson risk model with dependence under a multi-layer dividend strategy. Applied Mathematics Journal, Chinese University 26: 1-13. [CrossRef] 